\title{
Desarrollo de la Creatividad y el Talento desde las Primeras Edades. Componentes Curriculares de un Programa de Maestría en Educación
}

\author{
Noemí Suárez ${ }^{(1)}$, Karina E. Delgado(1), Isabel C. Pérez ${ }^{(2)}$ y María N. Barba(3) \\ (1) Centro de Investigaciones de Ciencias Humanas y de la Educación de la Universidad Tecnológica Indoamérica, \\ Calle Agramonte y Manuelita Sáez, Ambato, Ecuador. (e-mail: noemisuarez@uti.edu.ec; karinadelgado@uti.edu.ec) \\ (2) Unidad de Planeamiento Académico de la Universidad Estatal de Quevedo, Avenida Carlos J. Arosemena 38, \\ Quevedo, Ecuador (e-mail: iperez@uteq.edu.ec) \\ (3) Docente de la Facultada de Ciencias Humanas y de la Educación, Universidad Técnica de Ambato, \\ Ave Los Chasquis y Río Payamino, Ambato, Ecuador (e-mail: mn.barba@uta.edu.ec)
}

Recibido Mar. 12, 2019; Aceptado Abr. 29, 2019; Versión final Jun. 4, 2019, Publicado Dic. 2019

\section{Resumen}

Se analizan los componentes curriculares de un programa de maestría en educación con especialización en desarrollo de creatividad y el talento, a partir de un estudio secuencial en tres fases. Los métodos empleados incluyeron el análisis de contenido temático de artículos de revistas de corriente principal, trabajos de titulación de docentes graduados en maestría de Educación, encuesta a docentes, e informes públicos que aportan datos sobre formación docente. Los resultados muestran tres problemas principales: i) tendencias en estudios que justifican el desarrollo creativo desde bases fisiológicas, psicológicas y sociológicas; ii) necesidades teóricas y metodológicas de docentes en ejercicio; y iii) oferta formativa limitada a otras especializaciones. Se concluye que, la evidencia teórica y empírica aporta referentes en la construcción de los componentes del programa propuesto para el contexto ecuatoriano en estudio.

Palabras clave: creatividad; talento; educación; formación; posgrado

\section{Development of Creativity and Talent from Early Ages. Curricular Components of a Master's Program in Education}

\begin{abstract}
The curricular components of a master's program in education with specialization in the development of creativity and talent, from a sequential study divided in three phases are analysed. The methods used included the analysis of thematic content to articles in mainstream journals, graduate works of teachers graduated from a Master's Education program, surveys to teachers and public information and reports that provide data on teacher training. The results show three main problems: i) trends in studies that justify creative development from physiological, psychological and sociological bases; ii) the theoretical and methodological needs of current teachers; and iii) the formative offer limited to other specialisations. It is concluded that the theoretical and empirical evidence provides references in the construction of the components of the proposed program for the Ecuadorian context under study.
\end{abstract}

Keywords: creativity; talent; education; training; graduate degree 


\section{INTRODUCCIÓN}

La sociedad actual necesita niños, niñas, adolescentes y jóvenes reflexivos, críticos, con visión de futuro, iniciativa y confianza que enfrenten los problemas actuales de forma singularmente creativa en los contextos donde se desenvuelven. Los estudios sobre la creatividad de Sternberg y Lubart (1991) reconocen entre otros elementos psicológicos asociados al comportamiento creativo: la motivación profesional, la originalidad, la independencia cognoscitiva y la flexibilidad como cualidades o rasgos que caracterizan a las personas creativas. En la literatura este constructo puede encontrarse fluctuando como proceso, producto, resultado, situación, clima y potencialidad mediante el cual el individuo o grupo produce un producto novedoso y útil. Se llega a estas conclusiones luego de analizar aportes (libro, revisiones) que aborda diferentes definiciones de forma explícita (Runco, 2017).

Mitjáns (2013) resalta el valor de la transformación del proceso de enseñanza-aprendizaje en el contexto de un clima creativo entre maestros y alumnos. Su propuesta de Sistema Didáctico Integral para el desarrollo de la creatividad destaca el carácter sistémico y permanente de las acciones pedagógicas, desde las primeras edades por parte del docente. De las definiciones anteriores se interpreta a la creatividad y al talento como expresión de la personalidad en su función reguladora; determinada por factores extrapersonales como la situación social contextual en que está inmerso el sujeto, su historicidad y las posibilidades reales que tiene para incrementar sus motivaciones.

La creatividad es producto y resultado de un proceso realizado por el individuo influido por otros y las circunstancias que le rodean. Razones que obligan a pensar en las relaciones entre desarrollo de la creatividad y el talento; en la personalidad, en constante búsqueda de satisfacer la necesidad humana de autorrealización; y en la enseñanza creativa, que debe propiciar el docente, desde las primeras edades. De ahí el rol trascendental que cumple la educación en preparar a los individuos para esta tarea y en pro de la humanidad. En el contexto escolar, el desarrollo de los profesores, el entorno y el currículo desempeñan un papel fundamental en la potencialización de la creatividad y el talento. (Ucus y Acar, 2019).

La visión de progreso, desde el campo de la educación, incluye a todos los ámbitos del conocimiento; contrario a la idea de que solo las actividades culturales y/o artísticas son las que pueden desempeñar un papel decisivo. Ello impregna un carácter complejo, multi e interdisciplinario a su formación. El estudio de Hernández et al. (2015) lo confirma al abordar la problemática en función del desarrollo de la creatividad, como competencia genérica en los currículos por su importancia en el desarrollo no solo de la persona, sino en todas las áreas del conocimiento y sectores de la sociedad. También, Laar et al. (2018) consideraron como indicadores de la creatividad, el pensamiento crítico y la resolución de problemas, a través de un instrumento teórico que fue validado para medir habilidades digitales del siglo XXI y utilizar las Tecnologías de la Información y la Comunicación para generar ideas novedosas en las diferentes áreas del conocimiento.

El estudio de López-Martínez y Navarro-Lozano (2010) se basó en la aplicación del programa de Renzulli, con el Test de Pensamiento Creativo de Torrance y el Cuestionario de Creatividad en 90 niños, mientras que el de Garaigordobil y Berrueco (2011) se apoyó en el juego cooperativo creativo en 86 niños. Las investigaciones experimentales demostraron lo alcanzable de este propósito a partir del diseño, aplicación y evaluación de programas de desarrollo creativo en niños de Educación Primaria.

Sin embargo, la comprensión de la relación dialéctica entre enseñanza y aprendizaje -desde el conocimiento del docente de las bases teóricas de naturaleza psicopedagógica y didáctico-metodológica- es clave para el desarrollo dinámico de la personalidad creativa en la infancia. Muchas veces las dificultades presentadas en el aprendizaje son resultado de prácticas deficientes de enseñanza (Miralles et al., 2012) que se manifiestan en conocimientos, destrezas y competencias de los estudiantes para desempeñarse a lo largo de toda la vida.

El aprendizaje creativo exige un docente que lo personalice y una escuela más justa e inclusiva para desarrollar óptimamente las capacidades de las personas. Su diagnóstico debe hacerse con métodos alternativos que fortalezcan el sistema de valoraciones del logro de los estudiantes, en función una evaluación para el aprendizaje y no solamente del aprendizaje con escalas numéricas rígidas (Huapaya et al., 2012). Muchas veces la acción del docente no deja margen a la incertidumbre, ni a una programación que favorezca la enseñanza utilizando itinerarios de aprendizajes personalizados y respuestas individuales a las necesidades educativas. El desarrollo creativo y del talento no se desarrolla por sí solo porque abarcan una dimensión personal y social.

Actualmente se plantea un énfasis en la educación centrada en el alumno y su aprendizaje, sin embargo, el aprendizaje supone un proceso guiado desde la enseñanza del docente. Por tanto, la educación tiene el 
desafío de producir un proceso pedagógico desarrollador del pensamiento creativo en los alumnos para ampliar ideas, generar hipótesis y buscar alternativas de solución innovadoras. De ahí que no se desestima el valor didáctico metodológico de la base orientadora que debe propiciar el docente como forma de expresión de lo humano y lo personológico en el desarrollo de la creatividad y el talento. En este sentido, la formación especializada de los docentes de las primeras edades es clave porque figuran como protagonistas.

Llegado a este punto se entiende la relación que existe entre el aprendizaje creativo y la formación requerida en los docentes para lograrlo. Se han realizado estudios relacionados con las funciones y necesidades de los docentes para promover la construcción de entornos de aprendizajes creativos (Davies, et al., 2014); así como de los factores personales y ambientales que afectan a las prácticas de fomento de la creatividad de los docentes. (Chan y Yuen, 2014). El desempeño docente se ve afectado por factores de distinta naturaleza, en unos asociados a la propia personalidad del maestro, el autoritarismo, el conformismo, la rigidez, la inmediatez, la rutina y en otros por el cumplimiento excesivo de la normativa y el control por parte de los gestores, dependiendo de la dinámica en la que desarrollan su actividad profesional.

Las barreras por diversidad cultural, ausencia de desafíos y emociones positivas hacia el aprendizaje, la falta de reflexión sobre el conocimiento incide en la dificultad para desarrollar esta dimensión del ser humano. También influye la preparación desde el punto de vista teórico-conceptual, instrumental para realizar evaluaciones del talento, potencialidades creativas, las estrategias y condiciones para su desarrollo desde las primeras edades (Ribeiro,et.al, 2016). La realidad del profesorado ecuatoriano no se aleja de lo señalado en estos estudios.

Específicamente, la evidencia aportada en el estudio de necesidades realizado en docentes, cuya formación es de licenciados y bachilleres de centros representativos de la zona 3 situada en región Sierra confirma las debilidades teóricas y prácticas de los docentes, así como la ausencia de propuestas formativas con estos fines. Este resultado indica la necesidad de replantear la formación docente y delimitar el objetivo de esta investigación a proponer los componentes curriculares de un programa de maestría en educación con especialización en desarrollo de la creatividad y el talento desde las primeras edades.

\section{METODOLOGÍA}

Esta investigación se realiza con el objetivo de proponer los componentes curriculares de un programa de maestría en educación con especialización en desarrollo de la creatividad y el talento en tres fases de manera secuencial y de tipo exploratoria descriptiva. Acoge una metodología que combina la perspectiva cualitativa y cuantitativa. En la primera fase referida al análisis de contenido temático de las investigaciones relacionadas con la creatividad y el talento; el propósito es determinar las tendencias en cuanto al predominio de un tipo u otro de resultado presentado en diferentes contextos internacionales. Las fuentes seleccionadas fueron 18 artículos publicados en revistas de corriente principal en el periodo comprendido entre los años 2010 y 2019 en países de Europa, Asía, América del Norte y América del Sur.

En la segunda fase, de análisis de las necesidades formativas de los docentes, se aplicó una encuesta a una muestra probabilística de 300 profesores de Educación Básica calculada de una población de 1359 con un nivel de confianza del 95\%. Esta población incluye solo a docentes sin especialización posgraduada, que se encuentran ejerciendo la docencia en escuelas emblemáticas representativas de la zona 3 y localizadas en tres provincias de la sierra ecuatoriana (Cotopaxi. Tungurahua, Chimborazo). Estas instituciones educativas pertenecen al sector público, son dotadas con equipamiento e infraestructura para garantizar el aprendizaje de sus estudiantes; y los docentes acceden a través de concurso de méritos y oposición, por la convocatoria del programa Quiero Ser Maestro del Ministerio de Educación. Luego se seleccionaron aleatoriamente 100 docentes de cada centro.

La encuesta se construyó y piloteó con la participación de 30 estudiantes de maestría en educación que concluían estudios en la Universidad Tecnológica Indoamérica. A partir de la enumeración de las ventajas y limitaciones se obtuvieron datos que permitieron un proceso de categorización de las principales opiniones de los docentes, se contrastaron con la teoría y luego se estructuraron los contenidos a indagar en la muestra seleccionada.

El cálculo del coeficiente Alpha de Cronbach $(0,649)$, según las posibilidades del SPSS y los ajustes realizados durante el pilotaje, permitió organizar las preguntas de acuerdo con los siguientes ejes temáticos: 1) Datos personales referidos al nivel de formación. 2) Bases neuro-psicopedagógicas del aprendizaje, la creatividad y el talento. 3) Técnicas de diagnóstico en la determinación de los problemas de aprendizaje, la creatividad y el talento. 4) Diseño de estrategias, metodologías y ambientes en el proceso de enseñanzaaprendizaje que propicien desempeños creativos. 5) Aplicación de evaluaciones que midan desempeños 
creativos. 6) Gestión e intervención en equipos multidisciplinarios para la atención de los problemas de aprendizaje y el talento.

En la tercera fase, relacionada con el análisis de la formación recibida, se indagó en los datos publicados través de la página web de la Secretaría Nacional de Ciencia y Tecnología. Esta informa sobre la oferta académica de maestrías, especializaciones, doctorados por universidades de la zona 3 en el área de educación, el periodo de vigencia, modalidad del programa y los contenidos de la malla curricular. También se analizó el documento "Informes de rendición de cuentas de la coordinación Zonal 3"; cuyo contenido refleja datos relacionados con la cantidad de docentes, condición de acceso y formación que poseen, cuando inician sus labores; y los cursos de capacitación ofrecidos por el Ministerio de Educación anualmente para las escuelas públicas.

En esta misma fase se estudió el contenido temático de 852 trabajos de titulación, de quienes culminaron estudios de maestría en el área de educación en universidades de la zona en estudio, con la finalidad de conocer los temas y /o problemas abordados en los trabajos de titulación relacionados con el desarrollo de la creatividad y el talento. La información de cada una de estas fuentes fue complementando la justificación de la necesidad de la propuesta de los componentes curriculares del programa de maestría.

\section{RESULTADOS}

Los resultados de esta investigación se presentan en tres fases. La primera, describe el análisis del contenido temático de investigaciones relacionadas con la creatividad y el talento para caracterizar las tendencias de los tipos de resultados presentados en los estudios en países de Europa, Asia, América del Norte, América del Sur y América Central. La categorización resultó en tres rubros referidos a las aportaciones teóricas de modelos, teorías y conceptos relacionados al desarrollo de la creatividad y el talento, acciones didácticas y pedagógicas realizadas por los docentes en la práctica, y formas de profesionalización docente para el desarrollo de la creatividad y el talento.

La segunda fase muestra un análisis sobre las necesidades formativas de los docentes, obtenida a través de una encuesta, organizada en 6 categorías temáticas. La tercera fase presenta el análisis de la formación recibida desde la oferta académica de programas existentes en la zona 3 del Ecuador, los cursos de superación que ofrece el Ministerio de Educación, así como las temáticas investigadas en los trabajos de titulación de los estudiantes de maestría actuales. Luego se muestra la nueva propuesta de formación que incluye los componentes curriculares de una maestría en educación, con especialización en desarrollo de la creatividad y el talento desde las primeras edades.

\section{Fase 1: Análisis de investigaciones relacionadas con la creatividad y el talento}

De manera general se identifican al menos dieciocho estudios en diferentes países indicando la preocupación de la comunidad científica sobre el tema. Se presentan, según la categorización en los rubros presentados anteriormente.

Resultados del primer rubro (siete estudios): aportaciones teóricas de modelos, teorías y conceptos relacionados al desarrollo de la creatividad y el talento.

Las aportaciones teóricas de las comunicaciones encontradas en la literatura versan sobre los siguientes temas: las funciones cognitivas y estructuras cerebrales (López-Fernández y Llamas-Salguero, 2018); teorías integradas de la relación creatividad - personalidad (Romoa et al., 2017); el papel de las formas de apropiación de lo real en la imaginación y la capacidad crítica (Murrieta et al., 2015); los aportes de las neurociencias (Burgos y Osses, 2015) y la dirección del proceso de la actividad psíquica (Kamak et al., 2017). Otros estudios incursionan en la influencia de los entornos en los que el niño se desenvuelve y el valor de la aceptación familiar en el desarrollo creativo (Krumm et al., 2013); así como las percepciones de familiares sobre el empleo de entornos naturales y seguros para desarrollar los juegos (Wang et al., 2018).

Los estudios mostrados en la literatura, en relación a este primer grupo, muestran tendencia a justificar la bases fisiológicas de funcionamiento cerebral; las psicológicas a partir de las condiciones generadas por este funcionamiento; el papel de las emociones y los afectos; las percepciones de la actividad psíquica en general y la sociológica, desde la influencia de los grupos primarios como la familia, lo que relaciona la dimensión personal y social desde las bases de estas ciencias y su frontera como la neuropsicología.

Resultados del segundo rubro (seis estudios): acciones didácticas y pedagógicas realizadas por los docentes en la práctica. 
El análisis de contenido develó la marcada orientación hacia el desarrollo creativo y menos al talento desde las primeras edades. Es así que el empleo de diferentes formas como programas desarrolladores del pensamiento divergente (López-Martínez y Navarro-Lozano, 2010); juegos cooperativos creativos (Garaigordobil, y Berrueco, 2011); producciones escritas de relatos de ficción (Méndez y Ghitis, 2015); el uso de improvisaciones musicales (Daugeliene y Straksiene, 2017); de libros ficticios (Webb et al., 2019) y las narrativas colaborativas digitales demostraron su eficacia en el desarrollo creativo (De Moral et al., 2018). En este grupo la tendencia es proponer soluciones, tomando como base la Didáctica, cuyos recursos son utilizados por los educadores para crear climas creativos en los estudiantes.

Resultados del tercer rubro (cinco estudios): formas de profesionalización docente para el desarrollo de la creatividad y el talento.

Los estudios que indican el interés en la formación del profesorado en esta temática, lo hacen desde el reconocimiento de las percepciones de los docentes sobre la promoción de la educación creativa en la primera infancia (Chu-ying y Hui, 2012). Fueron realizados en docentes de Hong Kong, Shanghai y Taiwán; concluyeron que existe una asociación de la ecología de la enseñanza creativa con el aprendizaje creativo; y exponen las barreras entre los profesores de distinto grado de experiencia. Se obtuvo entre los veteranos una puntuación significativamente más alta en esta asociación comparada con los maestros a mitad de carrera y los maestros novatos. En ese mismo año se encontró otro estudio correspondiente a una experiencia de práctica formativa desarrollada en Irlanda, en paralelo con los programas de doctorado universitario de cualquier disciplina, integrando organizaciones externas en sus programas de enseñanza para acreditar docentes en Innovación (Jarvis y Mitchell, 2012).

El hallazgo más significativo fue que los socios empresariales encuentran ventajas al tener equipos de estudiantes provenientes de esta academia en su lugar de trabajo, pues los jóvenes revitalizan a su personal; aumenta la confianza, creatividad y habilidades del trabajo en equipo. Mitjáns (2013) en Brasil propone un Sistema Didáctico Integral que indica las acciones pedagógicas posibles a tomar por los docentes para un aprendizaje creativo. A la luz de las teorías más actuales sobre la formación inicial y continua como base para la compresión del fenómeno del aprendizaje y la creatividad. Burgos y Osses (2015) en Chile presentan un estudio teórico que enfatiza en la necesidad de incorporar los aportes de las neurociencias. El desarrollo de la competencia de la información e iniciativa creativa en estudiantes de posgrado en Rusia se logró a partir del uso de materiales de valor práctico (Tabachuk, et al, 2018). Este tercer grupo sitúa los resultados en estudios de percepción del docente, el uso de medios materiales, programas de enseñanza y en el trabajo didáctico-metodológico.

Concluyendo, los tres grupos de investigaciones presentadas enfatizan en enfoques teóricos y modelos para una mejor comprensión del tema relacionado con el desarrollo de la creatividad y el talento, las acciones pedagógicas que intentan desarrollarla desde el aula, y la formación inicial y continua del profesorado. Pero se ven carencias en resultados que muestran una influencia formativa, sistematizada e integral de las ciencias; que subyacen en los procesos creativos y de desarrollo del talento desde las bases teóricas y las prácticas didácticas en maestros, para su potenciación a partir de las primeras edades. No hay resultados que evalúen intervenciones en docente con este objetivo, impidiendo tomar decisiones sobre formas de desarrollo validadas en la práctica formativa del docente.

El hecho de que la temática aparece con frecuencia, como objeto de estudio de varias regiones sustentados en una u otra disciplina científica, indica primero: la existencia real de la problemática en el contexto educativo; y segundo, el carácter multi-intertransdisciplinar y complejo que encierra la comprensión de sus dimensiones. Otro aspecto relevante es la forma de materializar la teoría -como estrategia en la dirección del proceso de enseñanza aprendizaje- tomando en consideración los diferentes contextos socioculturales en los que se desenvuelve la formación de niños, adolescentes y jóvenes; lo cual genera tensiones y retos a la profesionalidad del maestro y evidencia la necesidad de su formación permanente. Al mismo tiempo delinea las pautas para estructurar el sistema de conocimientos y las metodologías apropiadas para la formación docente inicial y continua.

\section{Fase 2: Análisis de las necesidades formativas en docentes}

La entrada a las instituciones educativas seleccionadas se realizó previa autorización del Ministerio de Educación en la coordinación zonal 3. El contenido de la encuesta fue revisado por funcionarios de esta instancia, quienes también seleccionaron las instituciones y acompañaron a los investigadores del equipo a establecer el contacto con los rectores de las instituciones educativas y en la presentación de los objetivos de la investigación. Luego a la muestra seleccionada aleatoriamente se le comunicó los objetivos y se aplicó la encuesta a 100 docentes en cada institución. El interés se concentró en determinar en qué medida se 
manifiestan las necesidades en este segmento de docentes (graduados de tercer nivel y bachilleres que se encuentran ejerciendo la docencia). Los resultados se muestran en la figura 1.

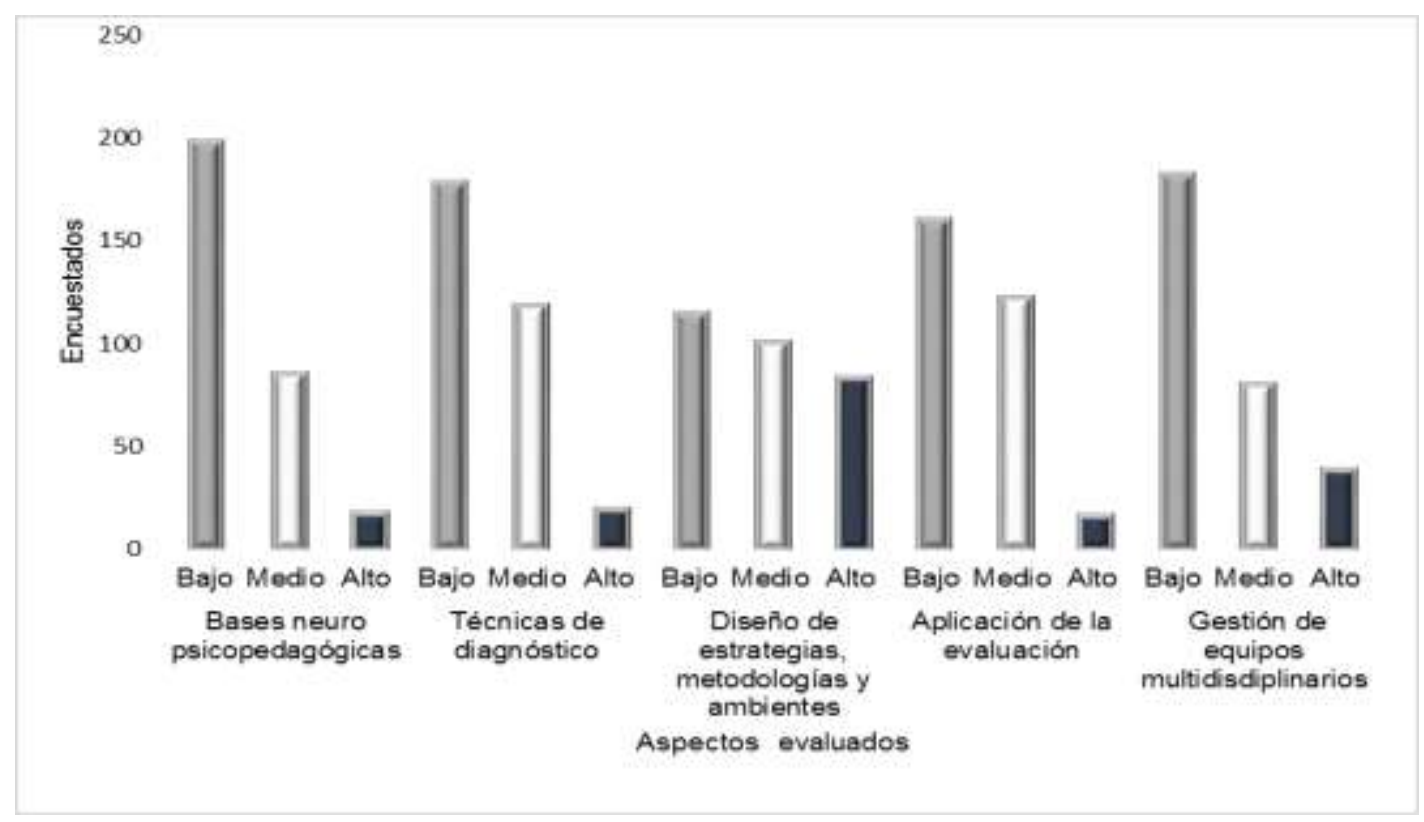

Fig. 1: Nivel de conocimiento de los docentes en la temática de desarrollo de la creatividad y el talento

Lo significativo de los datos encontrados es que un número alto de docentes, de la muestra en estudio que oscila entre 160 y 198, se califica de bajo en los conocimientos sobre las bases neuro-psicopedagógicas del aprendizaje, la creatividad y el talento; las técnicas de diagnóstico en la determinación de los problemas de aprendizaje, la creatividad y el talento; la aplicación de evaluaciones que midan desempeños creativos y en la gestión e intervención en equipos multidisciplinarios para la atención de los problemas de aprendizaje y el talento. Lo cual es comprensible desde la interdependencia entre cada uno de estos aspectos.

La ausencia de una teoría que aporte los criterios e indicadores para construir instrumentos, en función de diagnosticar estados iniciales, trae consigo fallas en el diseño de las evaluaciones de seguimiento al desarrollo creativo y fomento del talento. Estos aspectos también pudieran minimizar las posibilidades del debate y el trabajo en equipos multidisciplinarios, a pesar de la existencia de personas especializadas como psicólogos, psicopedagos y trabajadores sociales en las instituciones ecuatorianas o en los llamados Departamentos de Consejerías Estudiantiles (DECE); que están dedicados a la atención de las necesidades educativas especiales.

Las evaluaciones de alto en todos los casos no superan a 38 docentes, excepto en el caso del diseño de estrategias, metodologías y ambientes en el proceso de enseñanza-aprendizaje que propicien desempeños creativos. Aquí la apreciación de los encuestados es mucho más favorable, pues reportan una evaluación de bajo 115 docentes y de alto se evalúan 84, lo cual pudiera deberse al empleo de estrategias como el juego ampliamente difundido en la educación primaria (López-Martínez y Navarro-Lozano, 2010; Garaigordobil, y Berrueco, 2011), pero no se sabe si aplican las estrategias con suficiencia de conocimientos teóricos y de sus implicaciones. Los docentes que se evalúan de bajo son 115 y es significativo para la muestra en estudio porque cada uno ejerce la docencia en grupos de aproximadamente 35 y 40 estudiantes, que no están recibiendo una influencia formativa desarrolladora de la creatividad y el talento.

\section{Fase 3: Análisis de la formación recibida}

\section{Resultados del estudio de la oferta de programas y cursos de superación}

Cabe resaltar que desde la década del 90 este asunto fue tomando interés en la región. En tres países se han formado a los docentes a través de especializaciones para satisfacer esta demanda: Colombia, Venezuela, Perú, a través del Proyecto Pensamiento Reflexivo y Creatividad, impulsando varias investigaciones en esta área, en unidad con el Instituto Pedagógico Latinoamericano y Caribeño (IPLAC) en Cuba y la cátedra UNESCO. Se incluyó varios cursos en los currículos de formación de docentes, posgrados especializados y se creó la maestría en Educación con una línea en Investigación en Desarrollo de la Creatividad (Calzado y Rodríguez, 2006). Ecuador muestra insuficientes recursos formativos destinados a la especialización del profesorado en esta área. La página web de la Secretaría Nacional de 
Ciencia y Tecnología informa que, en la zona 3, se ofertan maestrías profesionalizantes como muestra la tabla 1, cuatro programas en la provincia de Tungurahua, en Chimborazo y Cotopaxi dos, y en Pastaza ninguno.

Tabla 1: Oferta académica de posgrados en el área de Educación zona 3, Ecuador

\begin{tabular}{|c|c|c|c|c|c|c|c|}
\hline Provincia & Educación & $\begin{array}{l}\text { Educación } \\
\text { Inicial }\end{array}$ & $\begin{array}{l}\text { Educación } \\
\text { Básica }\end{array}$ & $\begin{array}{l}\text { Innovación y } \\
\text { Liderazgo en } \\
\text { Educación }\end{array}$ & Pedagogía & $\begin{array}{l}\text { Tecnología e } \\
\text { Innovación } \\
\text { Educativa }\end{array}$ & $\begin{array}{l}\text { Pedagogía de } \\
\text { la Lengua y } \\
\text { Literatura }\end{array}$ \\
\hline Tungurahua & 1 & & & 1 & 1 & & 1 \\
\hline \multicolumn{8}{|l|}{ Pastaza } \\
\hline Chimborazo & & & & & 1 & 1 & \\
\hline Cotopaxi & & 1 & 1 & & & & \\
\hline
\end{tabular}

El análisis del currículo de estos programas, con vigencia desde 2014, descubre que son insuficientes para atender las necesidades formativas de los docentes en los procesos de inclusión de los niños, adolescentes y jóvenes con altas capacidades en el aprendizaje; y el desarrollo de la creatividad y el talento en el nivel Inicial y de Educación Básica. Específicamente las maestrías en Educación Inicial y Básica ponen énfasis en el desarrollo de las asignaturas de acuerdo a las áreas a impartir en el currículo de esos niveles educativos y así son estructuradas curricularmente. Las maestrías de Educación y Pedagogía mencionadas buscan formar en los conocimientos básicos de la docencia, pues se han concebido para docentes sin especialización posgraduada que se encuentran ejerciendo sin titulación de tercer nivel, procedentes de los programas "Quiero ser Maestro" del Ministerio de Educación, ante el déficit de docentes para cubrir la demanda; aunque pueden acceder también los titulados.

En el caso de las maestrías de Innovación, Liderazgo y Tecnología se ajustan a la necesidad de formar al docente en el conocimiento de las características de la innovación y las formas de liderar procesos nuevos en la escuela; así como en el uso de la tecnología, pero no en los procedimientos neurocognitivos y metodológicos que sirven de base al desarrollo creativo y estímulo del talento. Además de las limitaciones curriculares, la modalidad y el tiempo de duración restringe las posibilidades formativas; pues en todos los programas, excepto el de Innovación y Liderazgo, se desarrollan en la modalidad presencial durante tres periodos académicos intensivos, que incluyen horarios de hasta 6 horas durante la semana, lo que dificulta el acceso a los programas por desarrollarse en paralelo con la actividad profesional cotidiana. Las líneas de investigación declaradas explícitamente en los programas mencionados tampoco abordan la temática.

Otro dato complementario al estudio de necesidad fue el obtenido del informe de rendición de cuentas de la coordinación Zonal 3, presentado por Baes (2017), indicando la entrada a las escuelas de 3573 docentes; cuyo ingreso se da luego de rendir pruebas de conocimientos de las asignaturas, psicométricas y de personalidad; pero enfrentan la práctica sin ningún nivel de especialización pedagógica. De los datos personales solicitados, a través de la encuesta a docentes en 3 centros representativos de la región, se pudo conocer que el nivel profesional predominante en el $78 \%$ de la muestra es el de Licenciados en Educación.

Asimismo, los cursos de educación continua ofertados por el Ministerio de Educación, tanto en el histórico como para el año actual, versan en temas que no potencian el desarrollo creativo y el talento desde las primeras edades. El de Lingüística e Interculturalidad fue concebido para preparar a los docentes en la impartición de la asignatura de Lenguaje y Comunicación, en escuelas multiculturales (idioma kichwa, shuar y español) del Ecuador; Inglés como lengua extranjera y el de Legislación Educativa para conocer y concretar a nivel microcurricular la ley de educación vigente. Los cursos de Sensibilización respecto a la Discapacidad se imparten bajo la concepción de inclusión de niños con déficit y el de Prevención de Violencia con énfasis en vulnerabilidad.

Resultados del análisis de la presencia de la temática y/o problemas estudiados sobre la creatividad y talento en los trabajos de titulación de maestría y su validez.

La recopilación de datos, que fundamenta las necesidades formativas de los docentes, incluye también el análisis de la presencia de la temática y los problemas del desarrollo de la creatividad y el talento abordados en 852 trabajos de titulación en maestrías del área de educación. La muestra -tomada de la Universidad Tecnológica Indoamérica (UTI) y la Universidad Nacional de Chimborazo (UNACH) entre el 2014 y $2018-$ indica que solo $10(1,17 \%)$ se relacionan con el tema de creatividad; no se encontró referencia sobre el talento en ninguno de los trabajos de titulación analizados, tal como se muestra en la tabla 2. Otro hallazgo fue constatar la ausencia de procesos de validación de los resultados tanto en lo teórico como en lo 
práctico. Este resultado evidencia la poca importancia que tiene el tema desde la investigación en los programas de maestría que recientemente se han desarrollado.

Tabla 2: Trabajos de titulación relacionados con temas de creatividad y talento

\begin{tabular}{|c|c|c|c|}
\hline Temas del trabajo de titulación & Programa & Universidad & Año \\
\hline $\begin{array}{l}\text { Libro sensorial para el desarrollo de la creatividad en los niños } \\
\text { del prescolar de la unidad educativa Juan León Mera la Salle. }\end{array}$ & $\begin{array}{l}\text { Magíster en Ciencias de la } \\
\text { Educación, Mención } \\
\text { Innovación y Liderazgo } \\
\text { Educativo }\end{array}$ & UTI & 2018 \\
\hline $\begin{array}{l}\text { Estrategias lúdicas para el desarrollo de la creatividad en } \\
\text { niños de } 4 \text { a } 5 \text { años en la unidad educativa "Augusto Nicolás } \\
\text { Martínez". }\end{array}$ & $\begin{array}{l}\text { Magíster en Ciencias de la } \\
\text { Educación, Mención } \\
\text { Innovación y Liderazgo } \\
\text { Educativo }\end{array}$ & UTI & 2018 \\
\hline $\begin{array}{l}\text { La actividad física en el desarrollo del pensamiento creativo de } \\
\text { los niños de } 2 \text { a } 3 \text { años del CIBV "sonrisitas" de San José de } \\
\text { Macají, Licán, Riobamba, período } 2016 \text {. }\end{array}$ & $\begin{array}{l}\text { Maestría en Educación } \\
\text { Parvulario Mención Juego } \\
\text { Arte y Aprendizaje }\end{array}$ & UNACH & 2017 \\
\hline $\begin{array}{l}\text { Los cuentos pedagógicos en el desarrollo de la imaginación en } \\
\text { los niños y niñas del centro infantil del Buen Vivir "Francisco } \\
\text { Chiriboga" Riobamba. } 2016\end{array}$ & $\begin{array}{l}\text { Maestría en Educación } \\
\text { Parvulario Mención Juego } \\
\text { Arte y Aprendizaje }\end{array}$ & UNACH & 2017 \\
\hline $\begin{array}{l}\text { Diseño y aplicación de un manual de artes plásticas "Mary" } \\
\text { para desarrollar la creatividad en la iniciación de Lecto- } \\
\text { escritura de los niños y niñas de } 4 \text { años de edad del centro de } \\
\text { educación inicial "Inés Jiménez", parroquia matriz, cantón } \\
\text { Alausí, Provincia de Chimborazo. Periodo 2013-2014. }\end{array}$ & $\begin{array}{l}\text { Maestría en Educación } \\
\text { Parvulario Mención Juego } \\
\text { Arte y Aprendizaje }\end{array}$ & UNACH & 2017 \\
\hline $\begin{array}{l}\text { La gimnasia cerebral y la creatividad de los niños y las niñas } \\
\text { de } 4 \text { a } 5 \text { años de edad de la unidad educativa Luis a Martínez } \\
\text { del cantón Ambato provincia del Tungurahua. }\end{array}$ & $\begin{array}{l}\text { Maestría en Educación } \\
\text { Parvulario Mención Juego } \\
\text { Arte y Aprendizaje }\end{array}$ & UNACH & 2017 \\
\hline $\begin{array}{l}\text { Estrategias didácticas creativas y pensamiento lógico } \\
\text { matemático en niños y niñas de } 3 \text { años de la unidad educativa } \\
\text { "Bolívar". Ambato-Tungurahua. Período } 2016 \text {. }\end{array}$ & $\begin{array}{l}\text { Maestría en Educación } \\
\text { Parvulario Mención Juego } \\
\text { Arte y Aprendizaje }\end{array}$ & UNACH & 2017 \\
\hline $\begin{array}{l}\text { Los cuentos pedagógicos en el desarrollo de la imaginación en } \\
\text { los niños y niñas del centro infantil del Buen Vivir "Francisco } \\
\text { Chiriboga" Riobamba. } 2016 \text {. }\end{array}$ & $\begin{array}{l}\text { Maestría en Educación } \\
\text { Parvulario Mención Juego } \\
\text { Arte y Aprendizaje }\end{array}$ & UNACH & 2017 \\
\hline $\begin{array}{l}\text { Los títeres como estrategia didáctica en el pensamiento } \\
\text { creativo de niños y niñas de } 5 \text { años de edad de la escuela de } \\
\text { educación básica "Julio Enrique Fernández" de la parroquia } \\
\text { Izamba cantón Ambato, provincia de Tungurahua, durante el } \\
\text { año } 2015 \text {. }\end{array}$ & $\begin{array}{l}\text { Magíster en Ciencias de la } \\
\text { Educación, Mención } \\
\text { Educación Parvulario }\end{array}$ & UTI & 2014 \\
\hline $\begin{array}{l}\text { La aplicación de la teoría socio cultural de Lev Vygotsky en el } \\
\text { comportamiento de los niños/as de primer año de educación } \\
\text { básica, de la escuela "Agustín Albán" del cantón Pujilí, } \\
\text { provincia de Cotopaxi, en el año } 2014 \text {. }\end{array}$ & $\begin{array}{l}\text { Magíster en Ciencias de la } \\
\text { Educación, Mención } \\
\text { Educación Parvulario }\end{array}$ & UTI & 2014 \\
\hline
\end{tabular}

La estrategia de investigación seguida aportó datos de manera secuencial, cada una de las fases y fuentes fue clave para mostrar las tendencias en los tipos de aportaciones en las investigaciones sobre el tema y el gran desafío que supone desarrollar la creatividad y talento en el contexto áulico, cuando se tienen limitaciones formativas para lograrlo. Llegado a este punto, se entiende la pertinencia de la formación de docentes de Inicial y Básica en programas de maestría o especialización en esta temática para la zona 3 de Ecuador. En adelante se propone una forma de organizar esta especialización considerando los componentes curriculares.

Propuesta de los componentes curriculares del programa de Maestría en Educación: Mención Creatividad y Talento.

El estudio presentado orienta la generación de un programa de maestría, cuyo objetivo es la formación docente para la gestión de un proceso de enseñanza-aprendizaje desarrollador de la creatividad y el talento en los niños y niñas, basado en un enfoque inter-trans disciplinario de las Ciencias de la Educación. Este se concreta a través de la organización de módulos, en tres unidades en la malla curricular, como se muestra en la figura 2. La formación epistemológica incluye los contenidos teóricos básicos esenciales, que permiten la comprensión del aprendizaje, la enseñanza, la creatividad y el talento. Luego se prevén módulos que tienen el objetivo de proporcionar herramientas para materializar la teoría en la práctica en una formación profesional avanzada con un enfoque disciplinar, multi e interdisciplinar. Las habilidades investigativas se desarrollan en la unidad de titulación que inicia la malla en el periodo 1 hasta su cierre en el periodo 4 . No obstante, todo el desarrollo curricular tiene un enfoque en lo investigativo de manera transversal. 


\begin{tabular}{|c|c|c|c|}
\hline $\begin{array}{l}\text { Período } \\
\text { Académico }\end{array}$ & $\begin{array}{l}\text { Unidad Básica } \\
\text { Formación Epistemológica }\end{array}$ & $\begin{array}{l}\text { Unidad disciplinar, multi e } \\
\text { interdisciplinar } \\
\text { Formación profesional avanzada }\end{array}$ & $\begin{array}{l}\text { Unidad de titulación } \\
\text { Investigación Avanzada }\end{array}$ \\
\hline \multirow{3}{*}{1} & Teoría de la enseñanza & & $\begin{array}{l}\text { Metodología para el diseño } \\
\text { del trabajo de titulación }\end{array}$ \\
\hline & & Desarrollo curricular & \\
\hline & & Evaluación de la enseñanza & \\
\hline \multirow{3}{*}{2} & Teoría del aprendizaje & & $\begin{array}{l}\text { Diseño de la propuesta de } \\
\text { titulación }\end{array}$ \\
\hline & & Diversidad y Aprendizaje & \\
\hline & & Evaluación del aprendizaje & \\
\hline \multirow{2}{*}{3} & $\begin{array}{l}\text { Teorías de inteligencia } \\
\text { creatividad y el talento }\end{array}$ & & \\
\hline & $\begin{array}{l}\text { Métodos y técnicas de } \\
\text { diagnóstico de la } \\
\text { creatividad y el talento }\end{array}$ & & $\begin{array}{l}\text { Aplicación de la propuesta } \\
\text { de titulación. }\end{array}$ \\
\hline \multirow[t]{3}{*}{4} & & $\begin{array}{l}\text { Estrategias y ambientes } \\
\text { desarrolladores de la creatividad y } \\
\text { el talento }\end{array}$ & \\
\hline & & $\begin{array}{l}\text { Diseño y desarrollo de propuestas } \\
\text { de intervención psicopedagógica }\end{array}$ & \\
\hline & & & $\begin{array}{l}\text { Validación de la propuesta } \\
\text { de titulación y publicaciones }\end{array}$ \\
\hline
\end{tabular}

Fig. 2: Organización de las asignaturas en la malla curricular

La duración total del programa es de veinticuatro meses, en modalidad semipresencial, con la asistencia presencial de ocho horas cada semana los días sábados. Cada periodo incluye asignaturas de 48 horas que abarcan veintiún meses, quedando tres para ajustes y culminación del trabajo de titulación con los tutores. Esta organización toma en consideración la infraestructura tecnológica disponible en la universidad y las posibilidades reales de acceso de los docentes al programa de maestría por realizarse fuera de la jornada laboral.

Entre las principales metodologías para la formación del docente se ha considerado oportuno incluir las siguientes: a) Proveer a los maestrantes de múltiples perspectivas teóricas en el abordaje del objeto de las asignaturas y sus representaciones en la práctica (abordar la problemática del entorno físico y social del estudiante). b) Proporcionar el análisis de casos reales, relevantes, significativos y auténticos, que reflejen las complejidades del aprendizaje en cada situación. c) Ofrecer bases orientadoras para la actividad de construcción y no solo para la reproducción del conocimiento. d) Proveer de metodologías, herramientas y ambientes que incentiven el autoanálisis, la reflexión, la autoconciencia y la meta cognición. e) Promover una práctica reflexiva y crítica, a partir del análisis de problemáticas de donde proceden los maestrantes. f) Permitir la articulación del contenido con el contexto en la construcción del conocimiento, a través del trabajo en equipo, colaboración y experiencia de los participantes, organizados en pequeños grupos afines. g) Enfatizar en la resolución de problemas, las habilidades de pensamiento complejo de orden superior, desde las interrelaciones conceptuales y los aprendizajes interdisciplinarios. h) Orientar hacia la elaboración de productos para integrar el aprendizaje y la acción. i) Posibilitar la reflexión sobre las condiciones de generalización de los productos a otros ámbitos diferentes de los que dieron lugar a su elaboración. 
El programa prevé dos líneas fundamentales de estudio que utilizarán el modelo de investigación acción, pudiendo articular los enfoques cualitativos y cuantitativos.

Desarrollo de la creatividad: incluye el estudio de los modelos de aprendizaje activo, con el propósito de desarrollar las potencialidades creativas. Especial énfasis se debe hacer en la utilización de recursos didácticos estimuladores de la creatividad a partir del elemento motivacional (Mitjáns, 2013). Se incluyen entrenamientos para potenciar el pensamiento crítico, la fluidez, flexibilidad, originalidad y elaboración, como elementos esenciales para que el docente pueda replicar este proceder con sus estudiantes (Garaigordobil y Berrueco, 2011). También las metodologías, estrategias, sugerencias prácticas de actividades creativas y los ambientes de enseñanza en el contexto escolar (Mitjáns, 2013). Todos estos instrumentos deben estar rigurosamente programados, seleccionados y adaptados a las distintas áreas curriculares y a la edad de los estudiantes. Las formas de organización para aprender a aprender, de forma cooperada, autónoma, con uso de las tecnologías, deberán ser estudiadas; considerando la creatividad como cualidad humana resultante de la actividad de grupos, actores y sectores de la sociedad y al mismo tiempo transformador de estos.

Igualmente puede incluirse el estudio de los ambientes específicos que potencian la creatividad, como el apoyo en ambientes extraescolares, y en el entorno familiar, en el cual se ha de crear un clima favorable para que la capacidad creadora fluya fácilmente.

Desarrollo del talento: el contexto ambiental y la personalidad del educando son diversos por múltiples factores, por ello se trata de generar propuestas facilitadoras del progreso de todos los estudiantes que conviven en los grupos escolares, para contribuir a la construcción de una sociedad más sostenible, justa, solidaria e inclusiva de aquellos talentos abandonados desde el punto de vista pedagógico. De modo que los estudios sobre la identificación y selección de estudiantes con talentos académicos, su personalidad, motivaciones e inclusión social, así como las estrategias o programas para su atención en el contexto escolar y extraescolar constituyen objeto de investigación en esta sublínea (Ribeiro y Stoltz, 2015).

\section{DISCUSIÓN FINAL}

La base epistemológica, construida por estudiosos del tema de la creatividad y el talento, ha transitado por varios enfoques: desde el psicométrico, cognitivo, integrativo, pragmático hasta el humanistapersonológico; comprendiéndose la multidimensionalidad de este y las dificultades teóricas y metodológicas de los docentes para la práctica educativa. Cada uno ha contribuido, de forma fragmentada, en la solución a los problemas asociados al desarrollo de la creatividad y el talento en el ámbito educativo, pero de lo que se trata es de comprender su complejidad y carácter interdisciplinario. En la revisión de la literatura se muestra la tendencia en los tipos de resultados de naturaleza teórica práctica, sobre todo en las primeras edades y en la formación del profesorado; destacándose la manera parcelada en la que se ha incidido sobre este.

Varios países de la región han orientado la formación de los docentes hacia el desarrollo creativo a través de maestrías; sin embargo, esta iniciativa no ha sido sistematizada en las prácticas formativas en Ecuador. El estudio, realizado en la zona sierra del país, detectó necesidades que orientan acciones de perfeccionamiento profesional en función de las tendencias actuales de la investigación en creatividad y talento; así como de los nuevos escenarios en los que se desenvuelven los docentes para impactar de forma positiva en la sociedad. Para las universidades que realizaron el estudio -y se encuentran en un proceso de reforma permanente con el fin de ofertar programas que respondan a las necesidades del contexto educativo- un programa de maestría en Educación con una la malla curricular que articula las estrategias de formación, el modelo de investigación y sus líneas al objeto de la enseñanza-aprendizaje desarrolladora de la creatividad y el talento, constituye la apuesta de mejora de los investigadores.

No obstante, el estudio tiene como limitación la muestra seleccionada, si bien el criterio de nivel profesional es clave para los objetivos de la investigación, no se estratificó de manera tal que permitiera a ciencia cierta conocer las diferencias en los resultados de quienes tienen un título de licenciado en educación y de los que tienen uno diferente a este. Se intuye el sesgo que supone realizar una encuesta a una población de docentes que se expone con frecuencia a esta técnica de estudio, por diferentes motivos; e instituciones que esperan una utilización de la información y la devolución de los resultados muchas veces anulados por los investigadores, dejando a los participantes en la incertidumbre sobre su aporte en el estudio.

Desde el punto de vista curricular dos cuestiones son relevantes cuando se analizan posibles limitaciones: la primera, si bien la propuesta responde a las necesidades del contexto en estudio, la malla curricular sugerida podría compararse en cuanto a las áreas de formación con otras maestrías y especializaciones ofertadas en la zona en función de movilidad internacional de profesores, armonización del título que se obtenga e integración de la comunidad educativa ecuatoriana en la dinámica del conocimiento de la región. La segunda, dada por la ausencia de un criterio de expertos del área del conocimiento que evalúen la 
coherencia de cada uno de los componentes curriculares y resulte complementaria de los datos obtenidos en la primera y segunda fase del estudio.

\section{CONCLUSIONES}

De acuerdo al trabajo presentado y a los resultados obtenidos, se pueden plantear las siguientes conclusiones principales:

1.- Existen fortalezas desde las teorías que valoran la relación entre lo personal y social para el desarrollo de la creatividad y el talento en el contexto áulico, y los estudios empíricos que destacan el papel de la preparación del docente, muchas veces alejado desde las propias concepciones acerca su rol y la no provisión de contenidos y metodologías para desempeñarse eficientemente en atención a esta necesidad del estudiante.

2.- La limitada oferta formativa en docentes da lugar a propuestas que emergen del contexto y, sin ser consideras únicas, pueden contribuir a impactar de manera positiva en la resolución del problema del desarrollo del pensamiento creativo y el talento desde las primeras edades, evitando así que los jóvenes lleguen a niveles educativos superiores incapacitados para resolver problemas de forma novedosa en el contexto donde se desarrollan.

3.-Es importante hacer efectivas instancias de retroalimentación que reconozcan y reivindiquen el valioso aporte de los participantes. Por eso se ha construido una propuesta de maestría, ajustada a las necesidades detectadas en los docentes, susceptible de perfeccionamiento con la contribución de expertos y estudios regionales que permitan su movilidad académica.

\section{REFERENCIAS}

Báez, C.L., Ministerio de Educación. Informe de rendición de cuentas de la coordinación Zonal 3, acceso: 22 de diciembre de 2018), Ecuador (2017)

Burgos, C. G. y B. S. Osses, Creatividad y Neurociencia: Binomio Esencial en el Contexto Educativo, doi: 10.4067/S0034-98872015000700018, Rev Med Chile, 143(7), 948-949 (2015)

Calzado, D. y M. Rodríguez, Consideraciones sobre el programa de maestría en educación del Instituto Pedagógico Latinoamericano y Caribeño, acceso: 24 septiembre de 2018), Cuba (2006)

Chan, S. y M. Yuen, Personal and Environmental Factors Affecting Teachers' Creativity-fostering Practices in Hong Kong, doi.org/10.1016/j.tsc.2014.02.003, Thinking Skills and Creativity, 12, 69-77 (2014)

Chu-ying, C. y A. N.N. Hui, Creativity in Early Childhood Education: Teachers' perceptions in three Chinese societies, doi.org/10.1016/j.tsc.2010.02.002, Thinking Skills and Creativity, 5 (2), 49-60 (2010)

Daugèlienè, J. y D. Strakšienè, Development of student's musical creative thinking by applying musical improvisation, doi.org/10.15823/p.2017.07, Pedagogika, 125(1), 97-110 (2017)

Davies, D., D. Jindal-Snape y otros cinco autores, The Roles and Development Needs of Teachers to Promote Creativity: A Systematic Review of Literature, doi.org/10.1016/j.tate.2014.03.003, Teaching and Teacher Education, 41, 34-41 (2014)

Del Moral, M. E., L. Villalustre y M.R. Neira, Percepción Docente del Desarrollo Emocional y Creativo de los Escolares Derivado del Diseño Colaborativo de Digital Storytelling, doi: 10.5944/educXX1.20202, Educación XX1, 21(1), 345-374 (2018)

Garaigordobil, M. y L. Berrueco, Effects of a Play Program on Creative Thinking of Preschool Children, doi: 10.5209/rev_SJOP.2011.v14.n2.9, Spanish Journal of Psychology, 14(2), 608-618 (2011)

Hernández, I., J.C. Alvarado y S.M. Luna, Creatividad e Innovación: Competencias Genéricas o Transversales en la Formación Profesional, Revista Virtual Universidad Católica del Norte, ISSN: 0124-5821, 44, 135-151 (2015)

Huapaya, C., F. Lizarralde y G. Arona, Modelo Basado en Lógica Difusa para el Diagnóstico Cognitivo del Estudiante, doi: 10.4067/S0718-50062012000100003, Formación Universitaria, 5(1), 13-20 (2012).

Jarvis, S. y F. Mitchell, Ireland's New Innovation Academy: Academic-practitioner Partnerships in Action, doi.org/ 10.1108/14777281211272251, Development and Learning in Organizations: An International Journal, 26 (6), 9-13 (2012)

Kamak, A., E. Auelbekov y otros tres autores, Creative Development of Junior School Students in the Process of Fine art Training, ISSN: 1528-2651, Journal of Entrepreneurship Education(JEE), 20 (3),1-7 (2017)

Krumm, G., J. Vargas-Rubilar y S. Gullón, Estilos Parentales y Creatividad en Niños Escolarizados, doi:10.5027/psicoperspectivas-vol12-issue1-fulltext-223, Psicoperspectivas, 12(1), 161-182 (2013) 
Laar, E., A.J.A. Deursen, J.A.G Dijk y J. Haan, 21st-Century Digital Skills Instrument Aimed at Working Professionals: Conceptual Development and Empirical Validation, doi.org/10.1016/j.tele.2018.08.006, Telematics and Informatics, 35(2), 2103-2388 (2018)

López-Martínez, O. y J. Navarro-Lozano, Influencia de una Metodología Creativa en un Aula de Primaria, doi.org/10.30552/ejep.v3i1.47, European Journal of Education and Psychology (EJPP), 3 (1), $89-102$ (2010)

López V. y F. Llamas, Neuropsicología del Proceso Creativo. Un Enfoque Educativo, doi.org/10.5209/RCED, Revista Complutense de Educación, 29 (1), 113-712 (2018)

Méndez, M. A., T. Ghitis, La Creatividad: Un Proceso Cognitivo, Pilar de la Educación, doi.org/10.4067/S071807052015000200009, Estudios Pedagógicos, 41(2), 143-155 (2015)

Miralles, P., J. Maquillón, F. Hernández y A. García, Dificultades de las Prácticas Docentes de Innovación Educativa y Sugerencias para su Desarrollo, ISSN: 1575-0965, Revista Electrónica Interuniversitaria de Formación del Profesorado (REIFO), 15(1), 19-26 (2012)

Mitjáns, A., Aprendizaje Creativo: Desafíos para la Práctica Pedagógica, CS (Cali), ISSN: 2011-0324, (11), 311-341 (2013)

Murrieta, M. I., F. Covarrubias y M.G. Cruz, La Participación de la Creatividad en los Procesos de Apropiación de lo real, doi.org/10.4067/S0717-554X2015000200008, Cinta de Moebio, (53), 205-217 (2015)

Ribeiro, F. H., T. Stoltz y otros seis autores, Barriers to Creativity, Identification and Inclusion of Gifted Student, ISSN: 2151-4771, Creative Education, 7, 1899-1905 (2016).

Ribeiro, F. H. y T. Stoltz, Creative Educational Practices for Inclusion of Gifted Children, doi: 10.4236/ce.2014.510093, Creative Education, 5, 803-808 (2014)

Romoa, M., J. Sánchez-Ruiz y V. Alfonso-Benlliure, Creatividad y Personalidad a Través de Dominios: Una Revisión Crítica, doi.org/10.1016/j.anpsic.2017.04.003, Anuario de Psicología, 47, 57-69 (2017)

Runco, M. A., Comments on Where the Creativity Research Has Been and Where Is It Going, doi:10.1002/jocb.189, The Journal of Creative Behavior, 51(4), 308-313 (2017)

Sternberg, R. y T. Lubart, An Investment Theory of Creativity and its Development, doi.org/10.1159/000277029, Human Development, 34 (1), 1-31 (1991)

Tabachuk, N.P. y I.A. Ledovskikh, Information Competency and Creative Initiative of Personality and Their Manifestation in Activity, Journal of Social Studies Education Research (JSSER), ISSN: 1309-9108, 9 (1), 168-186 (2018)

Ucus, S. y I.H. Acar, Exploring the Perceptions of Student Teachers about 'Creative School' in Early Childhood Education, doi.org/10.1080/03004430.2017.1307838, Early Child Development and Care, 189(2),191-206 (2019)

Wang, X., H. Woolley y otros tres autores, Young Children's and Adults' Perceptions of Natural Play Spaces: A Case Study of Chengdu, Southwestern China, doi.org/10.1016/j.cities.2017.08.011, Cities, 72, 173-180 (2018)

Webb, A.N., K.S. Zhbanova y A.C. Rule, An Investigation of Whether Fantasy Books, Compared to Same Topic Nonfiction, Promote Second Graders' Creativity, doi.org/10.1080/03004279.2017.1409782, Education, 47(1),116-131 (2019) 